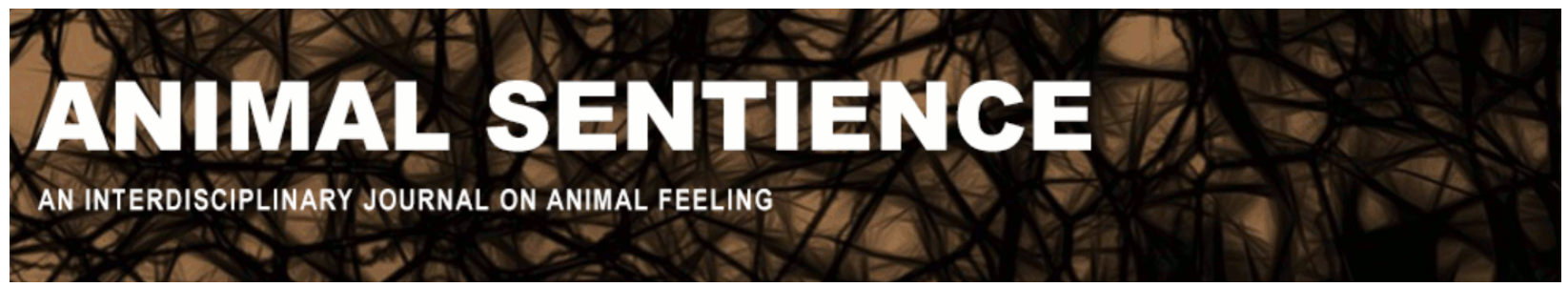

Zentall, Thomas R. (2017) The study of emotion in animals. Animal Sentience 14(3)

DOI: $10.51291 / 2377-7478.1227$

Date of submission: $2017-10-17$

Date of acceptance: 2017-10-18

(c)

This article has appeared in the journal Animal

Sentience, a peer-reviewed journal on animal

cognition and feeling. It has been made open access,

free for all, by WellBeing International and deposited

in the WBI Studies Repository. For more information,

please contact

wbisr-info@wellbeingintl.org.

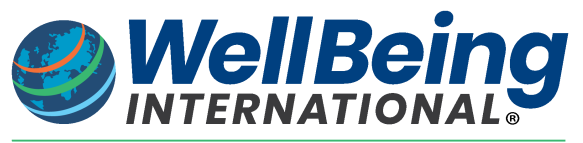

SOLUTIONS FOR PEOPLE, ANIMALS AND ENVIRONMENT 


\title{
The study of emotion in animals
}

Commentary on Kujala on Canine Emotions

\author{
Thomas R. Zentall \\ Department of Psychology \\ University of Kentucky
}

\begin{abstract}
The responsiveness of dogs to humans encourages us to attribute human-like emotions to them. Indirect evidence for emotions in other animals can be obtained but one must be careful to find means of distinguishing what we believe to be evidence for such emotions from simpler mechanisms. For example, is a dog's growl an indication of anger, fear, or possibly an unemotional defense of territory? By carefully designing experiments, we may be able to rule out alternative accounts and show better evidence for underlying emotions.
\end{abstract}

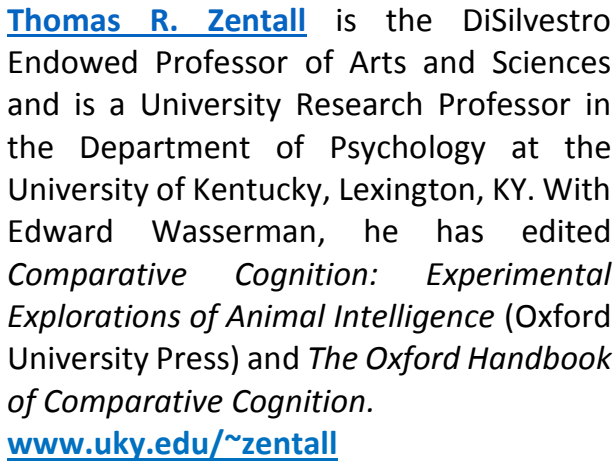

Thomas R. Zentall is the DiSilvestro Sciences University of Kentucky, Lexington, KY. With Edward Wasserman, he has edited Comparative Cognition: Experimental Explorations of Animal Intelligence (Oxford University Press) and The Oxford Handbook www.uky.edu/ zentall

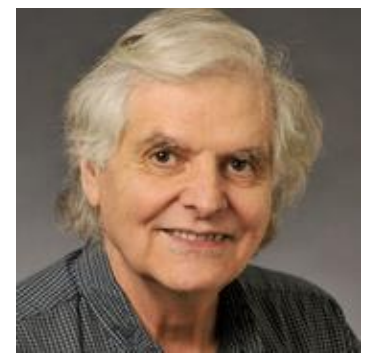

In her target article, Kujala (2017) presents a well-written and well-balanced discussion of the study of animal emotions focusing primarily on the emotions of dogs. On the one hand, she cites behavioral studies that interpret the behavior of dogs under conditions that would often elicit certain emotions in humans (e.g., happiness, sadness, surprise, fear, anger). On the other hand, she cites brain scan studies suggesting that under similar conditions, activity in the dogs' brain occurs in brain areas analogous to those of humans. The convergence of behavioral and brain data lends credibility to the possibility that the emotions may be similar as well. But as Kujala acknowledges, there is a strong tendency for humans to anthropomorphize about living and even nonliving things, so we must be careful to guard against attributing human emotions to animals when their behavior appears to us to indicate a similar emotion.

If one is interested in studying emotions in animals, dogs should provide a good model because not only are they naturally a highly social species, but through artificial selection over thousands of years they have become domesticated and integrated into our social network. Yet that also makes us more vulnerable to over-interpreting their behavior. Consider a dog's growl. Most people would interpret it as an indication of anger, but others may interpret it as an indication of fear, a quite different emotion, and still others may interpret it as a territorial display that may have little emotional content but simply be a signal to indicate "this is my territory." 
Secondary emotions are even more difficult to identify. Research on "guilt" described by Kujala is a good example. Although many pet owners claim that their dogs show signs of guilt, if they have engaged in forbidden behavior while the owner was away, Horowitz (2009) found that behavior, commonly thought to be a sign of "guilt," was evident when the guardians scolded their dog, regardless of whether the dog had disobeyed. This suggests that the dogs respond to the guardian's behavior rather than feeling guilt, due to remorse, based on their own behavior.

Another example of presumed secondary emotions described by Kujala is inequality aversion or having a sense of "fairness." When Range, Horn, Viranyi, and Huber (2009) trained dogs to present their paw on command, they did so less often when a partner dog was rewarded but they were not, than in an asocial condition when they were not rewarded. In such research it is important to rule out other possible explanations. One alternative is that when the partner was rewarded, a reward was present but not provided to the target dog. Would the target dog have done the same if, in the asocial condition, a reward was visible but not given to the dog when the paw was given?

In support of this alternative account, when monkeys that had been trained to exchange a stone for a low-valued reward saw a partner receiving a high-valued reward for performing the same action, they sometimes rejected the low-valued reward (Brosnan \& De Waal, 2003). However, when the same high-valued reward was placed in the adjoining cage without a conspecific partner, the target monkeys rejected the low-valued reward to a similar degree. Hence it is not necessary to posit that the monkey had a sense of fairness. Instead, the difference between the high-valued reward expected and the low-valued reward received could account for the monkeys' behavior. In the case of the dogs studied by Range et al. (2009), seeing the reward (whether given to the partner or not) may have been sufficient to reduce the likelihood of performing the requested behavior. In fairness to Kujala, she does mention that future research is needed to determine the role of other factors and expectation violation was one of the factors identified.

Kujala does a nice job of parsing the concept of empathy as an emotion in humans, noting differences among emotional empathy, cognitive empathy, and the separation of the self-fromthe-other. She notes that emotional empathy can be further divided into emotional contagion/self-distress and empathic concern. It is the latter difference that is critical to the attribution of the emotion. If dogs are thought to have a sense of empathy, it implies that they are concerned for the welfare of others and not merely that it relieves their own distress. To illustrate this difference, Lucke and Batson (1980), studying altruism in rats, included appropriate controls and found that the rats responded to the distress of other rats primarily to relieve their own distress. In more recent research, Bartal, Decety, and Mason (2011) found that rats would release a trapped conspecific from a tube, but they failed to consider the possibility that the "altruist" was motivated by affiliation with the trapped rat rather the desire to relieve the presumed distress of the other rat.

In research on altruism, it is important to ensure that the presumed altruist "understands" the nature of its behavior. For example, Kujala cites Quervel-Chaumette et al. (2015) who trained dogs to pull a rope attached to a tray to deliver food to an adjacent enclosure to which they could gain access. When the adjacent enclosure contained a familiar dog, they pulled the rope more than when the adjacent enclosure contained an unfamiliar dog. First, did the donor dog "understand" that they would not receive the delivered food itself? Second, could the presence 
of the unfamiliar dog in the adjacent enclosure inhibit pulling because of fear or reluctance to compete with the unfamiliar dog? In considering a process, like an emotion, that is difficult to assess directly, one must be careful to consider other simpler mechanisms.

The possibility of simpler alternative accounts is of particular concern when evaluating research mentioned by Kujala on the phenomenon known as the "cognitive judgement bias." This bias is purported to allow one to distinguish between whether an animal is optimistic or pessimistic. To test for an animal's bias, one might first train them to discriminate between a location that has a bowl with food and a location with one that does not (Mendl et al., 2010). Then one places a bowl at a location between the two training locations and assesses the time to approach the novel bowl location. If the dog approaches the test bowl quickly, it is judged to be optimistic. If they approach the test bowl slowly, they are judged to be pessimistic. The results of such a test are interesting. The problem with this test is the interpretation given to the results. Optimism and pessimism are emotions that relate to the expectation of specific future events, finding the bowl with or without food. It is possible, however, that differences in the latency of approach to the bowl in a novel location reflects individual differences in the dogs' fear of novelty. Kujala does note that inducing positive or negative expectations experimentally has proven tricky, but she does not suggest that the presumed emotions may be based on simpler mechanisms.

Many years ago, Morgan (1894) proposed that "In no case is an animal activity to be interpreted in terms of higher psychological processes if it can be fairly interpreted in terms of processes which stand lower in the scale of psychological evolution and development." The application of Morgan's Canon to research on comparative cognition is not intended as insistence on a simplistic interpretation of all behavior; it is intended to inquire whether the cognitive interpretation is necessary. When used appropriately, it is meant to encourage researchers to think of simpler explanations and by so doing, to consider how one would test the hypothesis that the simpler explanation is not adequate to account for the observed results. The process of ruling out alternative explanations for behavior is especially difficult when attempting to assess the emotions of an animal, but if we are to progress in our understanding of animal behavior, it is particularly important to heed Morgan's advice.

\section{References}

Bartal, B-A., Decety, J., \& Mason, P. (2011). Helping a cage-mate in need: empathy and prosocial behavior in rats. Science, 334, 1427-1430.

Brosnan, S. F., \& De Waal, F. B. (2003). Monkeys reject unequal pay. Nature, 425, 297-299. doi:10.1038/nature01963

Horowitz, A. (2009). Disambiguating the "guilty look": salient prompts to a familiar dog behaviour. Behavoural Processes, 81, 447-452. doi:10.1016/j.beproc.2009.03.014

Kujala, M. V. (2017). Canine emotions as seen through human social cognition. Animal Sentience 14(1).

Lucke, J. F., \& Batson, C. D. (1980). Response suppression to a distressed conspecific: Are laboratory rats altruistic? Journal of Experimental Social Psychology, 16, 214-227.

Mendl, M., Brooks, J., Basse, C., Burman, O., Paul, E., Blackwell, E., \& Casey, R. (2010). Dogs showing separation-related behaviour exhibit a 'pessimistic' cognitive bias. Current 
Biology, 20, R839-R840. doi:10.1016/j.cub.2010.08.030

Morgan, C. L. (1894). An Introduction to Comparative Psychology. London: Walter Scott.

Quervel-Chaumette, M., Dale, R., Marshall-Pescini, S., \& Range, F. (2015). Familiarity affects other-regarding preferences in pet dogs. Scientific Reports, 5, 18102. doi:10.1038/srep18102

Range, F., Horn, L., Viranyi, Z., \& Huber, L. (2009). The absence of reward induces inequity aversion in dogs. Proceedings of the National Academy of Sciences of the United States of America, 106, 340-345. doi:10.1073/pnas.0810957105 